\title{
Modular Properties of Algebraic Type Systems
}

\author{
Gilles Barthe ${ }^{1 \star}$ \\ gilles@cwi.nl
}

\author{
Herman Geuvers ${ }^{2,3}$ \\ hermanQwin.tue.nl
}

${ }^{1}$ CWI, Amsterdam, The Netherlands

${ }^{2}$ Faculty of Mathematics and Informatics, University of Nijmegen, The Netherlands

${ }^{3}$ Fac. of Math. and Informatics, Techn. Univ. of Eindhoven, The Netherlands

\begin{abstract}
We introduce the framework of algebraic type systems, a generalisation of pure type systems with higher order rewriting $\grave{a}$ la Jouannaud-Okada, and initiate a generic study of the modular properties of these systems. We give a general criterion for one system of this framework to be strongly normalising. As an application of our criterion, we recover all previous strong normalisation results for algebraic type systems.
\end{abstract}

\section{Introduction}

Algebraic-functional languages, introduced by Jouannaud and Okada in [19], are based on a very powerful paradigm combining type theory and higher-order rewriting systems. These languages embed in typed $\lambda$-calculi higher-order rewriting and hence allow the definition of abstract data types as it is done in equational languages such as OBJ. Examples of such languages which have been studied in the literature include the algebraic simply typed $\lambda$-calculus ([19]), algebraic type assignments systems ([2]) and the algebraic calculus of constructions ([3]). In this paper, we introduce a very general framework to study the combination of type theories with higher-order rewriting systems. The combination is based on pure type systems ([4]); the resulting framework of algebraic type systems covers in particular the systems of the algebraic $\lambda$-cube, a generalisation of Barendregt's cube studied in [3, 19]. A particular interest of algebraic type systems is to offer the possibility to initiate a generic study of the meta-theory of the combination between type theory and rewriting. First, basic meta-theoretic results, such as the substitution lemma or the generation lemma $([4,16])$ can be proved for arbitrary algebraic type systems. Second, one can address modularity results in a very abstract way, as it has been successfully done in term-rewriting (some striking examples can be found in $[21,26]$ ). The main contribution of this paper is to give a general criterion for an algebraic type system to be strongly normalising. As an application of our criterion, we obtain a new proof of the modularity of strong normalisation for the algebraic cube $([2,3,10,11,19]$ for

* This work was performed while working at the University of Nijmegen (The Netherlands) and visiting the University of Manchester (United Kingdom). 
subsystems). We also derive a strong normalisation result for algebraic higherorder logic (the algebraic extension of $\lambda H O L$ [16]) and the algebraic calculus of constructions with universes (with left-linear and confluent rewriting systems). In our view, the distinctive features of our approach are its generality (all the known results on modularity of termination for algebraic type systems can be obtained as a corollary of our result), its simplicity (the complexity of the proof is similar to the corresponding strong normalisation argument for pure type systems) and its flexibility (it is easy to adapt the proof to variants of pure type systems).

The paper is organised as follows: in the next section, we introduce algebraic type systems. In section 3 , we give an alternative syntax in which variables come labelled with a potential type and show the 'equivalence' between the two formulations. Besides we formulate a general criterion for an algebraic type system to be strongly normalising. In section 4, we prove strong normalisation for those systems satisfying the criterion by a general model construction. Section 5 focuses on the applications of the result to existing systems. The last section contains some final remarks about the work as well as directions for future research. We assume the reader to be reasonably familiar with pure type systems and their basic meta-theory, as presented for example in [15], [4] or [16].

\section{Combining higher-order rewriting systems and pure type systems}

\subsection{Higher-order rewriting systems}

In this section, we introduce higher-order rewriting systems. The framework we consider is slightly less general than the one of $[3,12,19]$ and has been chosen for clarity of presentation. For examples and applications of the general schema, the reader is refered to $[12,19]$.

Let $\Lambda$ be a set. Elements of $\Lambda$ are called base data ${ }^{2}$. The set of data is defined inductively as follows:

- every base datum is a datum;

- if $\sigma_{1}, \ldots, \sigma_{n}$ are data and $\tau$ is a base datum, then $\left(\sigma_{1}, \ldots, \sigma_{n}\right) \rightarrow \tau$ is a datum.

For convenience and without loss of generality, we can always assume the type of a function symbol to be of the form $\left(\sigma_{1}, \ldots, \sigma_{m}, \tau_{1}, \ldots, \tau_{n}\right) \rightarrow \tau_{n+1}$ where the $\sigma_{i}$ 's are data of arrow type and the $\tau_{i}$ 's are base data. Such data are called higher-order data. The set of first-order data is the subset of higher-order data for which $m=0$, i.e. a first-order datum is one of the form $\left(\tau_{1}, \ldots, \tau_{n}\right) \rightarrow \tau_{n+1}$ where the $\tau_{i}$ 's are base data. The set of higher-order data is denoted by $\Lambda^{*}$. When there is no risk of confusion, we will simply talk about data.

Definition $1 A$ higher-order signature $\Sigma$ over $\Lambda$ consists of an indexed family of (pairwise disjoint) sets $\left(\mathcal{F}_{w}\right)_{w \in \Lambda^{*}}$.

${ }^{2}$ Usually elements of $\Lambda$ are called sorts. We prefer to keep this name for the sorts of the pure type system. 
Elements of the $\mathcal{F}_{w}$ 's are called function symbols. A function symbol is first-order if it belongs to $\mathcal{F}_{w}$ for some first-order datum $w$ and higher-order otherwise. For every datum $\sigma$, the set $T_{(\Sigma, \sigma)}$ of terms of datum $\sigma$ is defined inductively. As usual, we start from a countably infinite set $V_{\sigma}$ for each datum $\sigma$. The rules are:

- elements of $V_{\sigma}$ are terms of datum $\sigma$;

- if $x \in V_{\left(\sigma_{1}, \ldots, \sigma_{n}\right) \rightarrow \tau}$ and $t_{i}$ has datum $\sigma_{i}$ for $i=1, \ldots, n$, then $x t_{1} \ldots t_{n}$ has datum $\tau$;

- if $f \in \mathcal{F}_{\left(\sigma_{1}, \ldots, \sigma_{n}\right) \rightarrow \tau}$ and $t_{i}$ has datum $\sigma_{i}$ for $i=1, \ldots, n$, then $f t_{1} \ldots t_{n}$ has datum $\tau$.

A term is first-order if all variables occurring in it are of base datum and all function symbols occurring in it are of first-order datum and is higher-order otherwise. In other words, first-order terms are of the form $f t_{1} \ldots t_{n}$ where $f$ is a first-order function symbol and the $t_{i}$ 's are first-order terms. Higher-order terms are of the form $F X_{1} \ldots X_{m} t_{1} \ldots t_{n}$ where the $X_{i}$ 's are higher-order variables and the $t_{i}$ 's are terms of base datum. Note that all terms are fully applied in the sense that only variables can be of higher-order datum ${ }^{3}$. The set var of variables of a term, occurences and substitutions are defined as usual.

Definition $2 A$ rewrite rule is a pair $(s, t)$ (written $s \rightarrow t$ ) of terms of the same datum such that $\operatorname{var}(t) \subseteq \operatorname{var}(s)$ and $s$ is not a variable. A rewrite rule is first-order if the terms are and higher-order otherwise.

In [19], Jouannaud and Okada define a general schema for higher-order rewrite rules.

Definition $3([3,19]) A$ higher-order rewrite rule $F X_{1} \ldots X_{m} t_{1} \ldots t_{n} \rightarrow$ $v$ satisfies the general schema if

1. F is a higher-order function symbol;

2. $F$ does not occur in any of the $t_{i}$ 's;

3. the higher-order variables occuring in the $t_{i}$ 's belong to $\left\{X_{1}, \ldots, X_{m}\right\}$;

4. for every subterm of $v$ of the form $F X_{1}^{\prime} \ldots X_{m}^{\prime} r_{1} \ldots r_{n}$, one has $\mathbf{t} \triangleright_{m u l} \mathbf{r}$ where $\triangleright_{\text {mul }}$ is the multiset extension of the strict subterm ordering.

Condition 3 is not essential but ensures that $F X_{1} \ldots X_{m} t_{1} \ldots, t_{n}$ is rewritable in the sense of [12]. Note that as a consequence of the definition, $F$ does not occur in any subterm of $v$ of the form $F X_{1}^{\prime} \ldots X_{m}^{\prime} r_{1} \ldots r_{n}$ except in head position. Higher-order rewrite rules are a mild generalisation of the rules of primitive recursion.

Definition $4 \mathrm{~A}$ higher-order rewriting system is a set of rewrite rules such that:

- first-order rules are non-duplicating ${ }^{4}$;

${ }^{3}$ Using fully applied terms is important if one wants to consider type systems with $\eta$-reduction, see [6].

${ }^{4}$ Recall that a rewrite rule $s \rightarrow t$ is non-duplicating if the number of occurences of each variable $x$ in $t$ is lesser or equal to the number of occurences of $x$ in $s$. 
- higher-order rules satisfy the general schema;

- there are no mutually recursive definitions of higher-order function symbols.

The last requirement is not essential but has been added to simplify proofs. In the sequel, we let $\rightarrow_{R}$ denote the algebraic reduction relation.

\subsection{Algebraic type systems}

In this paragraph, we extend the framework of pure type systems with higherorder rewriting $\dot{a}$ la Jouannaud-Okada. The resulting framework of algebraic type systems covers a large class of algebraic-functional languages and provides a suitable basis to study modular properties of these languages.

Definition 5 An algebraic type system (or apts for short) is specified by a quintuple $\lambda S=(\mathcal{R}, S$, sortax, rules, datax $)$ where

- $\mathcal{R}$ is a finite list of higher-order rewriting systems $\mathcal{R}_{i}=\left(\Lambda_{i}, \Sigma_{i}, R_{i}\right)^{5}$ for $i=1, \ldots, n$;

- $S$ is a set of sorts;

- sortax $: S \rightarrow S$, rules $: S \times S \rightarrow S$ are partial functions;

- datax $:\left\{\Lambda_{1}, \ldots, \Lambda_{n}\right\} \rightarrow S$ is a total function.

Note that the definition implicitely requires the algebraic type system to be functional in the sense of [16] (such systems are called singly-sorted in [4]). This is not a real restriction as one can hardly imagine a non-functional pure type system of interest.

Definition 6 Let $V$ be an arbitrary infinite set of variables. The set of pseudoterms Pseudo of an algebraic type system $\lambda \mathcal{S}=(\mathcal{R}, S$, sortax, rules, datax $)$ is defined as follows:

- elements of $V$, sorts and data are pseudo-terms;

- if $A, B$ are pseudo-terms and $x \in V$, then $A B, \lambda x: A . B$ and $\Pi x: A . B$ are pseudo-terms;

- if $f$ is a function symbol of some signature $\Sigma_{i}$ of datum $\left(\tau_{1}, \ldots, \tau_{n}\right) \rightarrow \tau$ and $t_{1}, \ldots, t_{n}$ are pseudo-terms, then $f t_{1} \cdots t_{n}$ is a pseudo-term.

There are two notions of reduction on pseudo-terms: algebraic reduction $\rightarrow_{R}$ inherited from the term-rewriting systems and $\beta$-reduction. The combined reduction is denoted by $\rightarrow_{m i x}$. The rules for derivation for $\lambda \mathcal{S}$ are:

\footnotetext{
$\overline{5}$ That is, $\Lambda_{i}$ is a set of (base) data, $\Sigma_{i}$ is a higher-order signature over $\Lambda_{i}$ and $\mathcal{R}_{i}$ is a higher-order rewriting system over $\Sigma_{i}$.
} 


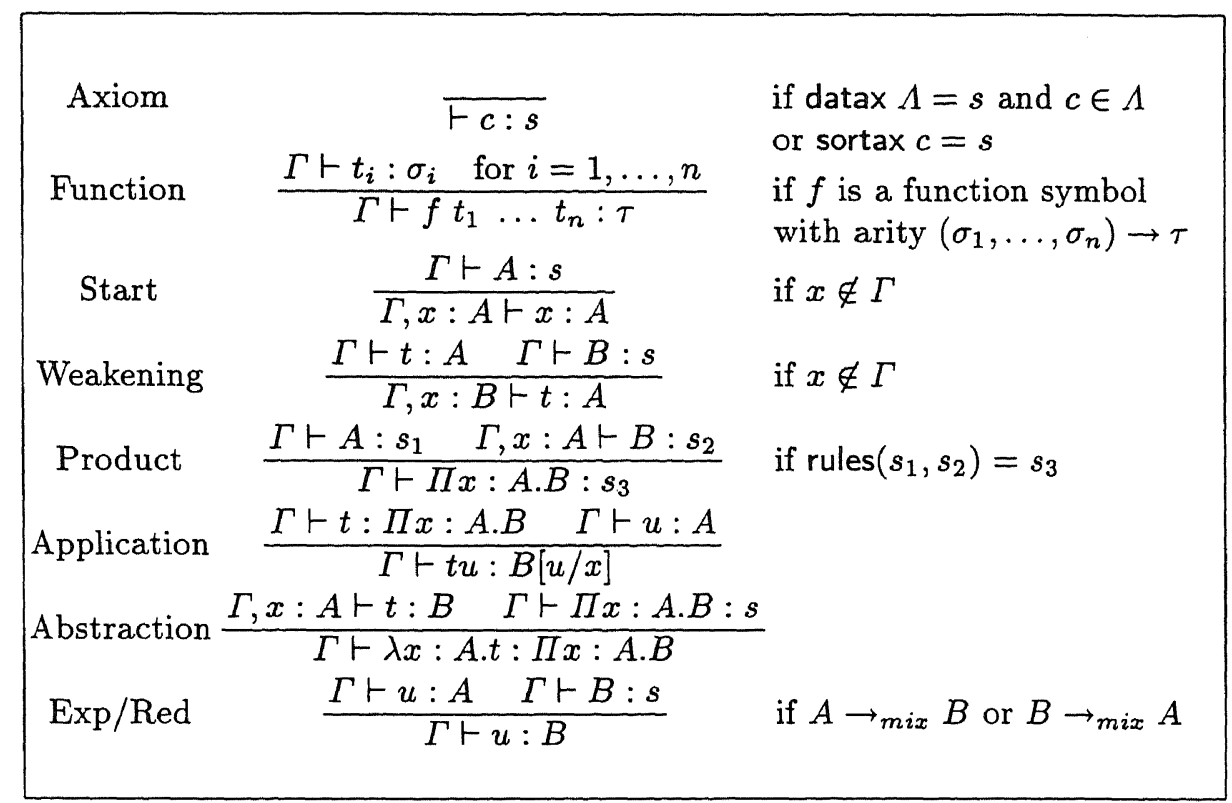

In an algebraic type system, the reduction relation is not confluent on the set of pseudo-terms; as a result, the usual proofs of subject reduction and of other results relying on subject reduction, such as strengthening cannot be extended. This motivates the following definition (see Section 6 for a longer discussion on subject reduction).

Definition 7 An algebraic type system $\lambda \mathcal{S}=(\mathcal{R}, S$, sortax, rules, datax $)$ has the subject reduction property if for all pseudo-terms $M, N, A$ with $M \rightarrow_{\beta} N$ and pseudo-context $\Gamma$,

$$
\Gamma \vdash M: A \quad \Rightarrow \quad \Gamma \vdash N: A
$$

As subject reduction for $R$-reduction holds in an arbitrary algebraic type system, it is easy to conclude that in an algebraic type system with the subject reduction property,

$$
\Gamma \vdash M: A \quad \Rightarrow \quad \Gamma \vdash N: A
$$

for every pseudo-context $\Gamma$ and all pseudo-terms $M, N, A$ with $M \rightarrow_{\text {mix }} N$.

Terminology For the sake of exposition, we conclude this paragraph by introducing some terminology.

Definition 8 An algebraic type system $\lambda \mathcal{S}=(\mathcal{R}, S$, sortax, rules, datax $)$ is $\mathcal{R}$ confluent (resp. $\mathcal{R}$-terminating, resp. $\mathcal{R}$-canonical, resp. $\mathcal{R}$-left-linear) if all its rewriting systems are confluent (resp. terminating, resp. canonical, resp. leftlinear).

In order to name algebraic type systems, it is useful to consider their underlying pure type systems. In the sequel, we will sometimes refer to an algebraic type system $\lambda \mathcal{S}=(\boldsymbol{R}, S$, sortax, rules, datax $)$ as an algebraic extension of the pure type system $\lambda \mathcal{S}^{\prime}=(S$, sortax, rules $)$. 


\section{A criterion for strong normalisation}

In [25], Terlouw gives a general criterion for a type system to be strongly normalising. We adapt his criterion to algebraic type systems and give an equivalent criterion in terms of algebraic type systems with labelled variables. The advantage of the second characterisation is that it eliminates the need to reason on contexts.

\subsection{Stratified algebraic type systems}

Recall that an environment is a family $\boldsymbol{\Gamma}=\left(x_{i}: A_{i}\right)_{i \in \mathrm{N}}$ where for every $i, x_{i}$ is a variable and $A_{i}$ is a pseudo-term such that for some sort $s_{i+1}, x_{0}: A_{0}, \ldots, x_{i}$ : $A_{i} \vdash A_{i+1}: s_{i+1}$.

Definition 9 Let $\Gamma=\left(x_{i}: A_{i}\right)_{i \in \mathrm{N}}$ be an environment.

- A pseudo-term $M$ is a prototype w.r.t $\Gamma$ if there exists a natural $i$, a sort $s$ and pseudo-terms $P_{1}, \ldots, P_{n}$ such that $x_{0}: A_{0}, \ldots, x_{i}: A_{i} \vdash M P_{1} \ldots P_{n}: s$.

- The relation $\prec_{\Gamma}$ on pseudo-terms is defined as the smallest relation such that for all $M, N \in$ Pseudo, if $M N$ is a prototype w.r.t. $\Gamma$, then $N \prec M$ and $M N \prec M$.

- An algebraic type system is stratified if the relation $\prec_{\Gamma}$ is well-founded for every environment $\boldsymbol{\Gamma}$.

The main result of the paper is the following criterion for strong normalisation.

Theorem 10 Every stratified $\mathcal{R}$-terminating algebraic type system with the subject reduction property is strongly normalising.

As a corollary, we recover the standard results on strong normalisation of algebraic type systems as well as some new results.

Corollary 11 - R-terminating extensions of systems of the $\lambda$-cube are strongly normalising ([3, 19]).

- R-terminating extensions of higher-order logic are strongly normalising.

- $\mathcal{R}$-canonical and $\mathcal{R}$-left-linear extensions of the algebraic calculus of constructions with universes are strongly normalising.

Note that for the first result, we use the fact that algebraic extensions of systems of the $\lambda$-cube have subject reduction ([3]). For the third result, note that leftlinearity of $\mathcal{R}$ (i.e. variables may only occur once in a left hand side of a rewrite rule) is a real restriction. However, there are interesting examples of higher-order rewrite rules that are left-linear, e.g.

$$
\begin{aligned}
\text { Maplist } X \text { nil } & \rightarrow_{R} \text { nil, } \\
\text { Maplist } X(\text { cons } a l) & \left.\rightarrow_{R} \text { cons }(X a) \text { (Maplist } X l\right) .
\end{aligned}
$$

The restriction to left-linearity is made, because if $\mathcal{R}$ is left-linear, then $\rightarrow_{\text {mix }}$ is confluent, hence we have the subject reduction property for the system and hence Theorem 10 applies. 


\subsection{Labelled variables}

In this section, we introduce a technical variant of (algebraic) type systems in which variables are "typed". This is reminiscent of some presentations of simply typed $\lambda$-calculus in which each type $\tau$ comes equipped with a set of variables of type $\tau$. In algebraic type systems, terms and types are defined simultaneously so the naive approach taken for simply typed $\lambda$-calculus cannot be used any longer. Our solution is to assign to every variable a pseudo-term, which will be its unique type if the variable is well-typed. In the sequel, we consider a fixed algebraic type system $\lambda S=(\mathcal{R}, S$, sortax, rules, datax $)$; as usual, its set of pseudo-terms is denoted by $T$.

Definition $12 A$ variable labelling is a map $\epsilon: V \rightarrow T$ such that the set $\{x \in V \mid \epsilon x=t\}$ is infinite for every $t \in T$.

Of course, such maps always exist if $V$ is sufficiently large (the cardinal of $V$ is determined by the cardinal of $S$ ). One nice aspect of variable labelling is that it eliminates the need to manipulate contexts. In the sequel, we assume we are given a fixed labelling $\epsilon$. We can define a notion of derivation w.r.t. $\epsilon$; the rules are

\begin{tabular}{|c|c|c|}
\hline Axiom & $\overline{F_{\epsilon} c: s}$ & if $\operatorname{datax} \Lambda=s$ and $c \in \Lambda$ or sortax $c=s$ \\
\hline Function & $\frac{\vdash_{\epsilon} t_{i}: \sigma_{i} \quad \text { for } i=1, \ldots, n}{\vdash_{\epsilon} f t_{1} \ldots t_{n}: \tau}$ & $\begin{array}{l}\text { if } f \text { is a function symbol } \\
\text { with arity }\left(\sigma_{1}, \ldots, \sigma_{n}\right) \rightarrow \tau\end{array}$ \\
\hline Start & $\frac{\vdash_{\epsilon} A: s}{\vdash_{\epsilon} x: A}$ & if $\epsilon x=A$ and $x$ is fresh in $A$ \\
\hline Product & $\frac{\vdash_{\epsilon} A: s_{1} \quad \vdash_{\epsilon} B: s_{2}}{\vdash_{\epsilon} \Pi x: A \cdot B: s_{3}}$ & if rules $\left(s_{1}, s_{2}\right)=s_{3}$ and $\epsilon x=A$ \\
\hline Application & $\frac{\vdash_{\epsilon} t: \Pi x: A \cdot B \quad \vdash_{\epsilon} u: A}{\vdash_{\epsilon} t u: B[u / x]}$ & \\
\hline Abstraction & $\frac{\vdash_{\epsilon} t: B \quad \vdash_{\epsilon} \Pi x: A . B: s}{\vdash_{\epsilon} \lambda x: A . t: \Pi x: A \cdot B}$ & \\
\hline Conversion & $\frac{\vdash_{\epsilon} u: A \quad \vdash_{\epsilon} B: s}{\vdash_{\epsilon} u: B}$ & if $A \rightarrow_{m i x} B$ or $B \rightarrow_{m i x} A$ \\
\hline
\end{tabular}

It is not difficult to check that algebraic type systems with variable labelling are essentially equivalent to algebraic type systems for systems with subject reduction.

Proposition 13 Assume the algebraic type system has subject reduction.

- If $\vdash_{\epsilon} M: A$, then $\Gamma \vdash M: A$ for some context $\Gamma$.

- If $\Gamma \vdash M: A$, then $\vdash_{\epsilon} \rho M: \rho A$ for some variable renaming $\rho$.

Proof sketch: The proof of the second part is by first renaming the bound and free variables in $\Gamma, M$ and $A$ in such a way that, if $x: B$ occurs in $\Gamma, M$ or 
$A$, then $\epsilon x=B$. The statement we obtain, say $\Gamma^{\prime} \vdash M^{\prime}: A^{\prime}$, is still derivable. Now one proves $\Gamma^{\prime} \vdash M^{\prime}: A^{\prime} \Rightarrow \vdash_{\epsilon} M^{\prime}: A^{\prime}$ by induction on the derivation.

For the proof of the first part, we first have to prove that, if subject reduction holds, then we have strengthening, which is the following property

$$
\text { If } \Gamma, x: A, \Delta \vdash M: B, x \notin \mathrm{FV}(\Delta, M, B) \text {, then } \Gamma, \Delta \vdash M: B \text {. }
$$

Using the fact that the underlying pure type system is functional, we can use the 'standard' proof (e.g. in [16]) prove strengthening. Using strengthening, one also proves a permutation property, which states the following.

$$
\text { If } \Gamma, x: A, y: C, \Delta \vdash M: B, x \notin \mathrm{FV}(C) \text {, then } \Gamma, y: C, x: A, \Delta \vdash M: B \text {. }
$$

Now, the following slight extension of the first result above can be proved by induction on the derivation (using strengthening and the permutation property).

$$
\begin{aligned}
\text { If } \vdash_{\epsilon} M: A, \text { then } & \Gamma \vdash M: A \\
& \text { for all } \Gamma \text { such that } \Gamma \text { respects } \epsilon \text { and } \operatorname{dom}(\Gamma)=\operatorname{FV}(M, A) .
\end{aligned}
$$

Here, $\Gamma\left(=x_{1}: C_{1}, \ldots, x_{n}: C_{n}\right)$ respects $\epsilon$ means that $\epsilon x_{i}=C_{i}$ and $x_{i+1} \notin$ $\mathrm{FV}\left(C_{1}, \ldots, C_{i}\right)$ for all $i$. Furthermore, $\operatorname{dom}(\Gamma)$ denotes the set $\left\{x_{1}, \ldots, x_{n}\right\}$.

It follows that strong normalisation and subject reduction of the system with labelled variables (or labelled system for short) is equivalent to strong normalisation and subject reduction of the original system. Besides, one can reformulate the criterion for systems with labelled variables.

Definition 14 Let $\lambda S$ be an algebraic type system with a variable labelling $\epsilon$. A prototype is a pseudo-term $M$ for which there exist $N_{1}, \ldots, N_{p} \in$ Pseudo and $s \in S$ such that

$$
\vdash_{\epsilon} M N_{1} \ldots N_{p}: s
$$

The set of prototypes is denoted by Proto. As before, we consider the relation $\prec$ defined as the smallest relation such that

$$
\forall M, N \in \text { Pseudo }[(M N) \in \text { Proto } \Rightarrow N \prec M \wedge(M N) \prec M]
$$

Definition $15 \lambda \mathcal{S}$ is stratified if the relation $\prec$ is well-founded.

Theorem 10 can now be rephrased as:

Theorem 16 Every $\mathcal{R}$-terminating stratified labelled type system with the subject reduction property is strongly normalising.

Theorem 10 follows easily from Theorem 16.

\section{The proof of the main theorem}

In this section, we prove Theorem 16. The proof is divided in two parts: in the first part, we prove that algebraic reduction is strongly normalising on legal terms. In the second part, we give a model-construction for stratified algebraic type systems. Strong normalisation is derived easily from the model construction. 


\subsection{Strong normalisation of algebraic reduction}

Strong normalisation of algebraic reduction on legal terms is established directly by advocating modularity results from [13] for example.

Proposition $17 \rightarrow_{R}$ is strongly normalising on legal terms.

Proof: the technique is inspired from [5] and consists of viewing $\lambda$-calculus as an algebraic signature. In this way, we define for every $\mathcal{R}$-algebraic type system $\lambda \mathcal{S}=(\mathcal{R}, S$, sortax, rules, datax $)$ an algebraic signature $\Sigma_{\lambda \mathcal{S}}$ extending the signatures of the rewrite systems and upon which algebraic reduction is terminating. Then we show that all legal terms can be obtained from the terms of $\Sigma_{\lambda \mathcal{S}}$ by an erasure map $\lceil$.$\rceil which reflects reduction. Strong normalisation of$ algebraic reduction on legal terms follows easily. In the sequel, we consider a finite sequence of terminating higher-order rewriting systems $\mathcal{R}_{i}=\left(\Lambda_{i}, \Sigma_{i}, R_{i}\right)$ for $i=1, \ldots, n$. Let $\Lambda=\bigcup_{i=1, \ldots, n} \Lambda_{i}$ and let $\Sigma_{\lambda S}=\left(\bigcup_{i=1, \ldots, n} \Sigma_{i}\right) \cup \Sigma_{0}$ where $\Sigma_{0}$ is the signature with function symbols:

$-\bar{s}_{\tau}: \tau$ for $s \in S$ and $\tau$ a datum,

- $\bar{\sigma}_{\tau}: \tau$ for $\sigma, \tau$ data,

- $\bar{\Pi}_{x, \tau_{1}, \tau_{2}, \tau_{3}}, \bar{\lambda}_{x, \tau_{1}, \tau_{2}, \tau_{3}}: \tau_{1} \times \tau_{2} \rightarrow \tau_{3}$ for every variable $x$ and $\tau_{1}, \tau_{2}, \tau_{3}$ data,

- Appl $\tau_{1}, \tau_{2}, \tau_{3}: \tau_{1} \times \tau_{2} \rightarrow \tau_{3}$ for every $\tau_{1}, \tau_{2}, \tau_{3}$ data.

The union $R_{0}$ of the $R_{i}$ 's is a higher-order rewriting system over $\Sigma_{\lambda \mathcal{S}}$. By hypothesis, its first-order reduction relation is terminating, so $R_{0}$ is terminating (see [13]).

To conclude the proof of the Proposition, first note that by subject reduction for $\rightarrow R$, we only need to prove that there is no infinite reduction through legal terms. To this end, we define a map from the terms of $\Sigma_{\lambda S}$ to pseudo-terms. For the sake of simplicity, we assume that the set of variables for every sort $\tau$ is $\left\{x^{\tau} \mid x \in V\right\}$. The map $\lceil$.$\rceil is defined as follows:$

$$
\begin{aligned}
\left\lceil x^{\tau}\right\rceil & =x \\
\left\lceil f\left(t_{1}, \ldots, t_{n}\right)\right\rceil & =f\left\lceil t_{1}\right\rceil \cdots\left\lceil t_{n}\right\rceil \\
\left\lceil\bar{\Pi}_{x, \tau_{1}, \tau_{2}, \tau_{3}}\left(t_{1}, t_{2}\right)\right\rceil & =\Pi x:\left\lceil t_{1}\right\rceil \cdot\left\lceil t_{2}\right\rceil \\
\left\lceil\bar{\lambda}_{x, \tau_{1}, \tau_{2}, \tau_{3}}\left(t_{1}, t_{2}\right)\right\rceil & =\lambda x:\left\lceil t_{1}\right\rceil \cdot\left\lceil t_{2}\right\rceil \\
\left\lceil\operatorname{Appl}_{\tau_{1}, \tau_{2}, \tau_{3}}\left(t_{1}, t_{2}\right)\right\rceil & =\left\lceil t_{1}\right\rceil\left\lceil t_{2}\right\rceil
\end{aligned}
$$

The map is surjective on the set of legal terms. Moreover, every infinite $R$ reduction sequence on legal terms can be lifted to an infinite $R_{0}$-reduction sequence on the terms of $\Sigma_{\lambda \mathcal{S}}$.

\subsection{The model construction}

In this section, we present a model construction for stratified algebraic type systems with the subject reduction property. The construction is based on saturated sets and is a generalisation of strong normalisation proofs for pure type systems, such as the polymorphic $\lambda$-calculus $([18,24,14])$ or the calculus of constructions $([17,25])$. The model is heavily inspired by [25]. Before giving a proof of Theorem 16, we need some preliminaries on saturated sets. 
Saturated sets Traditionally, saturated sets are defined as sets of $\beta$-strongly normalisable untyped $\lambda$-terms. Here we consider a slightly different notion of saturated sets, more adapted to our framework: we define saturated sets as sets of pseudo-terms rather than as sets of $\lambda$-terms. This is not really important but makes the proof slightly more elegant. Moreover, we consider typed saturated sets as in $[20,25]$ rather than untyped saturated sets. This means that the notion of saturated sets is defined relative to a set of pseudo-terms. This is not important for pure type systems but turns out to be crucial for algebraic type systems (otherwise, we cannot use the results of the principal case).

Recall that a pseudo-term $M$ is strongly normalising if all reduction sequences starting from $M$ are finite. The set of strongly normalising terms is denoted by SN. Saturated sets will be defined as subsets of SN with certain closure properties.

Definition $18 A$ base term is a term of the form $x P_{1} \ldots P_{n}$ where $x \in V$ and $P_{1}, \ldots, P_{n} \in \mathrm{SN}$.

The set of base terms is denoted by Base. Note that all base terms are strongly normalising.

Definition 19 Key-reduction $\rightarrow_{k}$ is the smallest relation on pseudo-terms such that for all pseudo-terms $M, N, O, P_{1}, \ldots, P_{n}$

$$
(\lambda x: M . N) O P_{1} \ldots P_{n} \rightarrow_{k} N[O / x] P_{1} \ldots P_{n}
$$

Note that a term has at most one key-redex. The term obtained from $M$ by contracting its key redex is denoted by $\operatorname{kred}(M)$.

Definition 20 Let $U \subseteq$ Pseudo. A set $X$ of pseudoterms is saturated in $U$ if

(i) $X \subseteq \mathrm{SN} \cap U$;

(ii) Base $\cap U \subseteq X$;

(iii) If $\operatorname{kred}(\bar{M}) \in X$ and $M \in \mathrm{SN} \cap U$, then $M \in X$.

The collection of all saturated sets in $U$ is denoted by $S A T(U)$. For $M \in$ Pseudo, we use $S A T(M)$ to denote the set of saturated sets in $\left\{N \in\right.$ Pseudo | $\left.\vdash_{\epsilon} N: M\right\}$. If $X \in \operatorname{SAT}(M)$, we say $X$ is a $M$-saturated set.

We list some closure properties of saturated sets.

Fact 21 Let $U, U^{\prime} \subseteq$ Pseudo.

- $\mathrm{SN}(U)=\mathrm{SN} \cap U$ is a saturated set in $U$.

- The set of saturated sets in $U$ is closed under arbitrary non-empty intersections.

- If $X$ is saturated in $U$ and $Y$ is saturated in $U^{\prime}$, then $X \rightarrow Y$ defined by

$$
X \rightarrow Y=\{M \in W \mid \forall N \in X . M N \in Y\}
$$

is saturated in $W$ provided that Base $\cap W \subset X \rightarrow Y$ (i.e. for every $w \in$ Base $\cap W$ and $x \in X, w x \in Y)$. 
- If $X$ is saturated in $U$ and $Y_{x}$ is saturated in $U_{x}^{\prime}$ for $x \in X$, then $\Pi x \in X . Y_{x}$ defined by

$$
\Pi x \in X . Y_{x}=\left\{M \in W \mid \forall N \in X . M N \in Y_{N}\right\}
$$

is saturated in $W$ provided Base $\cap W \subset \Pi x \in X . Y_{x}$ (i.e. for every $w \in$ Base $\cap W$ and $x \in X, w x \in Y_{x}$ ).

If $M \in$ Pseudo, then $\mathrm{SN}(M)$ is the saturated set of strongly normalising terms of type $M$.

The principal case The key fact in the model construction for algebraic type systems is that the sets of strongly normalising terms of base datum enjoy suitable closure properties.

Proposition 22 Let $f$ be a function symbol of datum $\left(\sigma_{1}, \ldots, \sigma_{n}\right) \rightarrow \tau$. Then for all pseudo-terms $t_{1}, \ldots, t_{n}$,

$$
t_{i} \in \mathrm{SN}\left(\sigma_{i}\right) \text { for } i=1, \ldots, n \Rightarrow f t_{1} \cdots t_{n} \in \mathrm{SN}(\tau)
$$

The proof is an adaptation of [19,2]. This key fact ensures that the model construction for algebraic type systems can be carried out in exactly the same way as for pure type systems.

Intuition behind the proof The idea of the proof is to give a model construction in which types are interpreted as (saturated) sets and legal terms as pseudo-terms such that the following soundness condition is satisfied:

$$
\vdash_{\epsilon} M: A \quad \Rightarrow \quad(M D \in\langle\langle A\rangle\rangle
$$

where $\langle\langle A\rangle\rangle$ is the saturated set interpretation of $A$ and (IMD is the pseudo-term interpretation of $M$. For simple systems, such as the (algebraic) simply typed $\lambda$-calculus $\lambda_{\rightarrow}$, the definition of $\langle\langle A\rangle\rangle$ can be given inductively on the structure of $A$ and the soundness condition can be proved by induction on the derivation. For the polymorphic $\lambda$-calculus $\lambda 2$, one is forced to parameterise interpretations by valuations. One then has to prove that if a valuation $\rho$ satisfies certain properties, then

$$
\vdash_{\epsilon} M: A \Rightarrow \llbracket M D_{\rho} \in\langle\langle A\rangle\rangle_{\rho}
$$

In a system with dependent types such as $\lambda P$ or $\lambda P \omega$, terms might occur in types so one cannot any longer define $\langle\langle A\rangle\rangle$ by induction on $A$. The standard solution is to define $\langle\langle A\rangle\rangle$ as a partial interpretation and show that it is well-defined on legal types. This requires the introduction of a new interpretation $a(M)$ which assigns to a term its possible values. (In this context valuations are of the form $(\rho, \zeta)$ where $\rho$ assigns to every variable (in some domain) a pseudo-term and $\zeta$ assigns to every variable (in some domain) a saturated set.) The idea is that $a(M)$ should be defined for every type $M$ and be a set of saturated sets such that under suitable conditions

$$
\vdash_{\epsilon} M: s \quad \Rightarrow \quad\langle\langle M\rangle\rangle_{\rho, \zeta} \in a(M)
$$


Here we see that dependent types introduce a new difficulty: we have indexed families of types, i.e. terms of type $B \rightarrow *^{6}$. These terms, which we have defined earlier as prototypes, will also need to be intepreted. To interpret them as families of types, we use induction on their structure: if $M$ is of type $B \rightarrow C \rightarrow *$, we want to define $a(M)$ as the set of families of maps $\left\{\left(f_{b}\right)_{b: B} \mid f_{b}: a(b) \rightarrow a(M b)\right\}$. This requires $a(b)$ and $a(M b)$ to be already defined. This requirement matches exactly the definition of $\prec$ : the assumption that $\prec$ is well-founded enables us to define the interpretation $a(M)$ by $\prec$-induction. The other two interpretations will be defined as usual by induction on the structure of the terms.

Convention From now on, we drop the subscript in $\vdash_{\epsilon}$.

The construction The set Data of data is defined as the union of the set of base data of the rewriting systems. The set Type of types is defined by

$$
\text { Type }=\{M \in \text { Pseudo } \mid \vdash M: s \text { for some } s \in S\}
$$

Definition 23 The map a: Pseudo $\rightarrow$ Set is defined by case distinction as follows.

- if $M \in$ Type $\backslash$ Data, $a(M)=\operatorname{SAT}(M)$;

- if $M \in$ Proto, $a(M)=\left\{\left(f_{B}\right)_{B \in \operatorname{cone}(M)} \mid f_{B}: a(B) \rightarrow a(M B)\right\}$;

- if $M \in$ Data, $a(M)=\{\mathrm{SN}(M)\}$;

- otherwise, $a(M)=\{\{\emptyset\}\}$;

where cone $(M)=\{B \in$ Pseudo $\mid(M B) \in$ Proto $\}$. Define $\mathbf{A}=\bigcup_{M \in \text { Pseudo }} a(M)$.

Definition $24 A$ valuation is a pair $(\rho, \zeta)$ such that $\rho: V \rightarrow$ Pseudo and $\zeta$ : $V \rightarrow \mathbf{A}$.

The extension (I. $D_{\rho}:$ Pseudo $\rightarrow$ Pseudo of $\rho$ is defined as the unique captureavoiding substitution extending $\rho$.

Definition 25 The map $\zeta$ is extended to terms by defining a map $\left\langle\langle.\rangle_{\rho \zeta}\right.$ : Pseudo $\rightarrow$ A as follows.

$$
\begin{aligned}
& \left\langle\langle x\rangle_{\rho \zeta}=\zeta(x)\right. \\
& \left\langle\langle\Pi x: A . B\rangle_{\rho \zeta}=\left\{P \in \operatorname{Pseudo} \mid \forall(N, Q) \in \mathcal{E}_{\rho \zeta}(A) .\right.\right. \\
& \text { if } x \in V, \rho(x) \in \text { Proto } \\
& \begin{array}{cc}
\left.\left.\langle\langle M N\rangle\rangle_{\rho \zeta}=\left(\langle\langle M\rangle\rangle_{\rho \zeta}\right)_{\left(N D_{\rho}\right.}\langle\langle N\rangle\rangle_{\rho \zeta} \quad P N \in\langle\langle B\rangle\rangle_{\rho(x:=N), \zeta(x:=Q)}\right)\right\} & \text { if }\left(\left[M x: A . B D_{\rho} \in T y P\right.\right. \\
\text { if }\left(\mid M N D_{\rho} \in\right. \text { Proto }
\end{array} \\
& \langle\langle\lambda x: A . b\rangle\rangle_{\rho \zeta}=\left(\boldsymbol{\lambda} c \in a(B) \cdot\langle\langle b\rangle\rangle_{\rho(x:=B), \zeta(x:=c)}\right)_{B \in \operatorname{cone}\left(\left[\lambda x: A . b D_{\rho}\right)\right.} \text { if }\left(\mid \lambda x: A . b D_{\rho} \in\right. \text { Proto } \\
& \left\langle\langle M\rangle_{\rho \zeta}=\mathrm{SN}(\mathrm{M})\right. \\
& \left\langle\langle M\rangle_{\rho \zeta}=\{\emptyset\}\right. \\
& \text { if } M \in \mathrm{Data} \\
& \text { otherwise }
\end{aligned}
$$

where for every $M \in$ Pseudo,

$$
\mathcal{E}_{\rho \zeta}(M)=\left\{(N, Q) \in \text { Pseudo } \times \mathbf{A} \mid \vdash N:\left(\mid M D_{\rho}, N \in\langle\langle M\rangle\rangle_{\rho \zeta}, Q \in a(N)\right\}\right.
$$

\footnotetext{
${ }^{6}$ This is not only true for dependent types but also for higher-order polymorphism as
it occurs in $\lambda \omega$.
} 

M.

The following lemma is easily established by induction on the structure of

Lemma 26 Let $M, N \in$ Pseudo. Let $(\rho, \zeta)$ and $\left(\rho^{\prime}, \zeta^{\prime}\right)$ be two valuations.

- If $\rho x=\rho^{\prime} x$ and $\zeta x=\zeta^{\prime} x$ for every $x \in \mathrm{FV}(M)$, then $\langle\langle M\rangle\rangle_{\rho \zeta}=\langle\langle M\rangle\rangle_{\rho^{\prime} \zeta^{\prime}}$.

- $\langle\langle M[N / x]\rangle\rangle_{\rho \zeta}=\left\langle\langle M\rangle_{\rho\left(x:=\left(\left[N D_{\rho}\right), \zeta\left(x:=\left\langle\langle N\rangle_{\rho \zeta}\right)\right.\right.\right.}\right.$

As a consequence of Lemma 26 and of the subject reduction property, we conclude that $\langle\langle.\rangle\rangle_{\rho \zeta}$ is invariant under reduction on legal terms.

Corollary 27 For every valuation $(\rho, \zeta)$ and terms $M, N$ such that $M \rightarrow_{\text {mix }} N$ and $\left\langle M D_{\rho},\left[N D_{\rho} \in\right.\right.$ Proto, we have $\langle\langle M\rangle\rangle_{\rho \zeta}=\left\langle\langle N\rangle_{\rho \zeta}\right.$.

In order to prove the main theorem, we must establish that the model behaves as expected. It requires a standard soundness argument. In the sequel, we call a context a finite list of variables $\Delta=y_{1}, \ldots, y_{n}$ such that for $i=1, \ldots, n$, $y_{i} \notin \mathrm{FV}\left(\epsilon y_{j}\right)(\forall j \leq i)$. One can check that for every term $M, \mathrm{FV}(M)$ can be ordered into a context.

Definition 28 Let $\Delta$ be a context. A valuation $(\rho, \zeta)$ satisfies $\Delta$ (notation $(\rho, \zeta) \models$ $\Delta)$ if for every $x \in \Delta$,

(i) $\vdash \rho x:\left([\epsilon x)_{\rho}\right.$,

(ii) $\rho x \in\langle\langle\epsilon x\rangle\rangle_{\rho \zeta}$,

(iii) $\langle\langle x\rangle\rangle_{\rho \zeta} \in a\left(\left(\left\langle x D_{\rho}\right)\right.\right.$.

We say that $\models M: A$ if for every valuation $(\rho, \zeta)$ satisfying $\mathrm{FV}(M) \cup \mathrm{FV}(A)$,

(i) $\vdash\left(M M D_{\rho}:\left(\left[A D_{\rho}\right.\right.\right.$,

(ii) $\| M D_{\rho} \in\left\langle\langle A\rangle_{\rho \zeta}\right.$,

(iii) $\left\langle\langle M\rangle_{\rho \zeta} \in a\left(\left\langle M D_{\rho}\right)\right.\right.$,

Fact 29 Let $(\rho, \zeta)$ be a valuation satisfying $\Delta$. Let $x \notin \Delta$ and $x \notin \mathrm{FV}(\epsilon y)$ for all $y \in \Delta$. Then for every $C \in a(x), \rho(x:=x), \zeta(x:=C)$ satisfies $\Delta \cup\{x\}$.

As $a(x) \neq \emptyset$, valuations can always be extended to a larger context while preserving satisfaction. We can now prove the main technical result of this paper.

Proposition 30 (Soundness) $\vdash M: A \Rightarrow \models M: A$.

Proof: by induction on the length of derivations.

- Axiom: if $\vdash s_{1}: s_{2}$ is an axiom, then it is easy to show $\models s_{1}: s_{2}$.

- Start: assume $\vdash x: A$ is deduced from $\vdash A: s$ by a start rule. Then $\epsilon x=A$. Assume $(\rho, \zeta)$ satisifies $\mathrm{FV}(A) \cup\{x\}$. By definition of satisfaction, $\vdash \rho x:\left(\mid A \rrbracket_{\rho}\right.$, $\rho x \in\langle\langle A\rangle\rangle_{\rho \zeta}$ and $\left\langle\langle x\rangle_{\rho \zeta} \in a(\rho x)\right.$, so we are done. 
- Function symbol: assume $\vdash f t_{1} \cdots t_{n}: \tau$ is deduced by a function rule from $\vdash$ $t_{i}: \sigma_{i}$ for $i=1, \ldots, n$ where $f$ is a function symbol of datum $\left(\sigma_{1}, \ldots, \sigma_{n}\right) \rightarrow$ $\tau$. Assume $(\rho, \zeta) \vDash F V\left(f t_{1} \cdots t_{n}\right)$.

$\vdash\left(f t_{1} \cdots t_{n} D_{\rho}: \tau\right.$ follows immediately from the induction hypothesis.

Next one has to prove that $\left(\left[f t_{1} \cdots t_{n} \rrbracket_{\rho} \in\left\langle\langle\tau\rangle_{\rho \zeta}\right.\right.\right.$. This is an immediate consequence of Lemma 22.

Finally, we need to prove $\left\langle\left\langle f t_{1} \cdots t_{n}\right\rangle_{\rho \zeta} \in a\left(\left(\left[f t_{1} \cdots t_{n}\right\rangle_{\rho}\right)\right.\right.$. This is easy because $\left(\left[f t_{1} \cdots t_{n} \rrbracket_{\rho} \notin\right.\right.$ Proto.

- Product: assume $\vdash \Pi x: A . B: s_{3}$ is deduced by a formation rule from $\vdash A: s_{1}$ and $\vdash B: s_{2}$. Let $(\rho, \zeta)$ be a valuation such that $(\rho, \zeta) \models \mathrm{FV}(\Pi x: A . B)$.

We prove $\vdash\left(\Pi \Pi x: A . B \rrbracket_{\rho}: s_{3}\right.$. By induction hypothesis, $\vdash\left(A \rrbracket_{\rho}: s_{1}\right.$. By fact 29 ,

$$
\rho(x:=x), \zeta(x:=C) \vDash \mathrm{FV}(\Pi x: A \cdot B) \cup\{x\}
$$

for every $C \in a(x)$. Hence $\vdash\left(B D_{\rho,(x:=x)}: s_{2}\right.$ by induction hypothesis. By the product rule, $\vdash \Pi x:\left([A)_{\rho} \cdot(B B)_{\rho,(x:=x)}: s_{3}\right.$. As $\Pi x:\left([A)_{\rho} \cdot\left([B)_{\rho,(x:=x)}=\right.\right.$ $\left(\Pi \Pi x: A \cdot B D_{\rho}\right.$, we conclude (i) holds.

Next we show $\left(\left[\Pi x: A . B D_{\rho} \in\left\langle\left\langle s_{3}\right\rangle_{\rho \zeta}\right.\right.\right.$. By definition of $\left\langle\langle\cdot\rangle_{\rho \zeta}\right.$, it is equivalent to show that $(\Pi \Pi x: A . B)_{\rho}$ is strongly normalising (we already know that (i) holds). By induction hypothesis, $\left[\mid A D_{\rho} \in\left\langle\left\langle s_{1}\right\rangle_{\rho \zeta} \subseteq \mathrm{SN}\right.\right.$ and $\left(\left[B D_{\rho^{\prime}} \in\right.\right.$ $\left\langle\left\langle s_{2}\right\rangle\right\rangle_{\rho^{\prime} \zeta^{\prime}} \subseteq \mathrm{SN}$ for every valuation $\left(\rho^{\prime}, \zeta^{\prime}\right)$ satisfying $\mathrm{FV}(B)$. Let $C \in a(x)$. Then $\rho(x:=x), \zeta(x:=C) \vDash \mathrm{FV}(\Pi x: A . B) \cup\{x\}$. Hence $\left[B D_{\rho(x:=x)} \in \mathrm{SN}\right.$ and $\left(I I x: A . B D_{\rho} \in \mathrm{SN}\right.$.

Finally, we show $\left\langle\langle\Pi x: A . B\rangle_{\rho \zeta} \in a\left(\left(\left[\Pi x: A . B D_{\rho}\right)\right.\right.\right.$. By (i), we know that $\left(\left[\Pi x: A . B D_{\rho} \in\right.\right.$ Type, so we have to prove that $\left\langle\langle\Pi x: A . B\rangle_{\rho \zeta}\right.$ is a

$\left(\Pi \Pi x: A . B D_{\rho}\right.$-saturated set. As $\left[A \rrbracket_{\rho}\right.$ is a type, it follows by induction hypothesis that $\left\langle\langle A\rangle_{\rho \zeta}\right.$ is a $\left(\left[A D_{\rho}\right.\right.$-saturated set. Besides, $\left\langle B D_{\rho(x:=x)}\right.$ is a type and by the substitution lemma, $(B)_{\rho(x:=N)}$ is a type whenever $\vdash N: \epsilon x$.

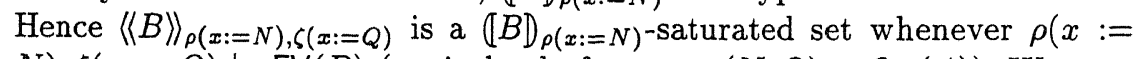
$N), \zeta(x:=Q) \vDash F V(B)$ (equivalently for every $\left.(N, Q) \in \mathcal{E}_{\rho \zeta}(A)\right)$. We conclude $\left\langle\langle\Pi x: A . B\rangle_{\rho \zeta}\right.$ is a $\left(\Pi \Pi x: A . B D_{\rho}\right.$-saturated set.

- Application: assume $\vdash M N: B[N / x]$ is deduced from $\vdash M: \Pi x: A . B$ and $\vdash N: A$ by an application rule. Let $(\rho, \zeta)$ be a valuation satisfying $\mathrm{FV}(M) \cup \mathrm{FV}(B[N / x])$.

First, we show that $\vdash\left(M N D_{\rho}:\left[B[N / x] D_{\rho}\right.\right.$. Consider the valuation $\left(\rho^{\prime}, \zeta^{\prime}\right)$ defined by

$$
\rho^{\prime} y=\left\{\begin{array}{l}
\rho y \text { if } y \in \mathrm{FV}(M) \cup \mathrm{FV}(B[N / x]) \\
y \text { otherwise }
\end{array}\right.
$$

and

$$
\zeta^{\prime} y=\left\{\begin{array}{l}
\zeta y \text { if } y \in \mathrm{FV}(M) \cup \mathrm{FV}(B[N / x]) \\
C_{y} \text { otherwise }
\end{array}\right.
$$

where $C_{y}$ is an arbitrary element of $a(y)$. Then

$$
\left(\rho^{\prime}, \zeta^{\prime}\right) \vDash \mathrm{FV}(M N) \cup \mathrm{FV}(\Pi x: A . B)
$$

By induction hypothesis, we have 
- $-\left(M M D_{\rho^{\prime}}:\left(\left[\Pi x: A \cdot B D_{\rho^{\prime}}\right.\right.\right.$

$-\vdash\left(\left[N D_{\rho^{\prime}}:\left(\left[A D_{\rho^{\prime}}\right.\right.\right.\right.$.

Hence $\vdash\left(\left[M N D_{\rho^{\prime}}:\left(B B D_{\rho^{\prime},(x:=x)}\left[\left(\left[N D_{\rho^{\prime}} / x\right]\right.\right.\right.\right.\right.$. In other words, $\vdash\left(M M N D_{\rho^{\prime}}\right.$ : $\left(B[N / x] \rho_{\rho^{\prime}}\right.$. As $\rho$ and $\rho^{\prime}$ coincide on $\mathrm{FV}(M) \cup \mathrm{FV}(B[N / x])$, we conclude that (i) holds.

Next, we show that $\left[M N D_{\rho} \in\left\langle\langle B[N / x]\rangle_{\rho \zeta}\right.\right.$. Note that it is equivalent to show $\left[M N D_{\rho^{\prime}} \in\langle\langle B[N / x]\rangle\rangle_{\rho^{\prime} \zeta^{\prime}}\right.$ where $\left(\rho^{\prime}, \zeta^{\prime}\right)$ is defined as above. By induction hypothesis, we know that $\vdash\left(\left[N D_{\rho^{\prime}}:\left(\left[A D_{\rho^{\prime}},\left[N D_{\rho^{\prime}} \in\left\langle\langle A\rangle_{\rho^{\prime} \zeta^{\prime}}\right.\right.\right.\right.\right.\right.$ and $\langle\langle N\rangle\rangle_{\rho^{\prime} \zeta^{\prime}} \in a\left(\left(\left[N D_{\rho^{\prime}}\right)\right.\right.$. Hence, $\left(\left[N D_{\rho^{\prime}},\langle\langle N\rangle\rangle_{\rho^{\prime} \zeta^{\prime}}\right) \in \mathcal{E}_{\rho^{\prime} \zeta^{\prime}}(A)\right.$. By induction hypothesis, $\| M D_{\rho^{\prime}} \in\left\langle\langle\Pi x: A . B\rangle_{\rho^{\prime} \zeta^{\prime}}\right.$. Hence

$$
\left(\left[M N D_{\rho^{\prime}} \in\langle\langle B\rangle\rangle_{\rho^{\prime}\left(x:=\llbracket \| N D_{\rho^{\prime}}\right), \zeta^{\prime}\left(x:=\left\langle\langle N\rangle_{\rho^{\prime} \zeta^{\prime}}\right)\right.}\right.\right.
$$

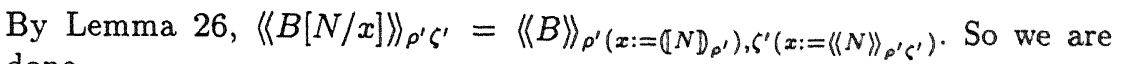
done.

Finally, we prove that $\left\langle\langle M N\rangle_{\rho \varsigma} \in a\left(\left(\left[M N D_{\rho}\right)\right.\right.\right.$. There are two cases two distinguish. If $\left[M N D_{\rho} \notin\right.$ Proto, then $a\left(\left(\left[M N D_{\rho}\right)=\{\{\emptyset\}\}\right.\right.$ and $\left\langle\langle M N\rangle_{\rho \zeta}=\right.$ $\{\emptyset\}$, so we are done. Otherwise, $\left[M D_{\rho} \in\right.$ Proto. By induction hypothesis, $\left\langle\langle M\rangle_{\rho \zeta} \in a\left(\left\langle M D_{\rho}\right)\right.\right.$ and $\left\langle\langle N\rangle_{\rho \zeta} \in a\left(\left(\left\langle N D_{\rho}\right)\right.\right.\right.$. Hence $\left(\left\langle\langle M\rangle_{\rho \zeta}\right)_{\left(N_{N} D_{\rho}\right.}\left\langle\langle N\rangle_{\rho \zeta} \epsilon\right.\right.$ $a\left(\left(M N D_{\rho}\right)\right.$.

- abstraction: assume $\vdash \lambda x: A . t: \Pi x: A . B$ is deduced by an abstraction rule from $\vdash t: B$ and $\vdash \Pi x: A . B: s$. Let $(\rho, \zeta)$ be a valuation satisfying $\mathrm{FV}(\lambda x: A . t) \cup \mathrm{FV}(\Pi x: A . B)$. We prove $\vdash(\lambda \lambda x: A . t)_{\rho}:\left(\Pi \pi x: A . B D_{\rho}\right.$.

By induction hypothesis, $\vdash\left(\Pi \Pi x: A \cdot B D_{\rho}: s\right.$. By Fact 29 we find that $\rho(x:=$ $x), \zeta(x:=C) \vDash \mathrm{FV}(t)$ for every $C \in a(x)$. Hence $\vdash(t)_{\rho(x:=x)}:\left(A D_{\rho(x:=x)}\right.$. As $x$ is not free in $A$, we have $\llbracket A \rrbracket_{\rho(x:=x)}=\llbracket A \rrbracket_{\rho}$. We can apply the abstraction rule to conclude.

Next we prove that $\left(\mid \lambda x: A . t D_{\rho} \in\left\langle\langle\Pi x: A . B\rangle_{\rho \zeta}\right.\right.$. This amounts to showing that for every $(N, Q) \in \mathcal{E}_{\rho \zeta}(A)$, we have

$$
(\| \lambda x: A . t\rangle_{\rho} N \in\left\langle\langle B\rangle_{\rho(x:=N), \zeta(x:=Q)}\right.
$$

By definition of saturated sets, this follows from

$$
(t)_{\rho(x:=N)} \in\left\langle\langle B\rangle_{\rho(x:=N), \zeta(x:=Q)}\right.
$$

which is a direct consequence of the induction hypothesis.

Finally we prove $\langle\langle\lambda x: A . t\rangle\rangle_{\rho \zeta} \in a\left([\lambda \lambda x: A . t\rangle_{\rho}\right)$. There are two cases to distinguish. If $\left([\lambda x: A . t)_{\rho} \notin\right.$ Proto, this is an easy consequence of the definitions. Otherwise, we have to prove that for every $B \in \operatorname{cone}\left(\left(\left\langle\lambda x: A . t D_{\rho}\right)\right.\right.$ and $c \in a(B),\left\langle\langle t\rangle_{\rho(x:=B), \zeta(x:=c)} \in a\left(\left(\mid \lambda x: A . t \rrbracket_{\rho} B\right)\right.\right.$. By the generation lemma, it follows that $\vdash B:\left([A)_{\rho}\right.$, hence $(\rho(x:=B), \zeta(x:=c))$ satisfies $\operatorname{FV}(t)$. The result is a consequence of the induction hypothesis.

- expansion/reduction: assume $\vdash M: B$ is deduced from $\vdash M: A$ and $\vdash$ $B: s$ using the expansion/reduction rule. Let $(\rho, \zeta)$ be a valuation satisfying $\mathrm{FV}(M) \cup \mathrm{FV}(B)$. As before, we can extend the valuation into a new valuation $\left(\rho^{\prime}, \zeta^{\prime}\right)$ such that $\left(\rho^{\prime}, \zeta^{\prime}\right)$ satisfies $\mathrm{FV}(M) \cup \mathrm{FV}(B) \cup \mathrm{FV}(A)$ and coincides with $(\rho, \zeta)$ on $\mathrm{FV}(M) \cup \mathrm{FV}(B)$. 
To prove $\vdash\left(\left[M D_{\rho^{\prime}}:\left([B)_{\rho^{\prime}}\right.\right.\right.$, note that $\left([A]_{\rho^{\prime}} \rightarrow\left([B)_{\rho^{\prime}}\right.\right.$ or $\left[(B)_{\rho^{\prime}} \rightarrow\left(\left[B D_{\rho^{\prime}}\right.\right.\right.$. Besides, it follows from the induction hypothesis that:

$-\vdash\left([M)_{\rho^{\prime}}:\left([A)_{\rho^{\prime}}\right.\right.$;

- $\vdash(B)_{\rho^{\prime}}: s$.

We conclude by the conversion rule.

To prove $\left[\mid M D_{\rho} \in\left\langle\langle B\rangle_{\rho \zeta}\right.\right.$, we just apply Corollary 27 .

Finally, $\left\langle\langle M\rangle_{\rho \zeta} \in a\left(\left(\mid M D_{\rho}\right)\right.\right.$ is immediate from the induction hypothesis.

Corollary $31 \vdash M: A \Rightarrow M \in \mathrm{SN}$.

Proof: for every derivation $\vdash M: A$, consider the valuation $(\rho, \zeta)$ such that $\rho(x)=x$ for every $x \in V$ and $\zeta(x)=\max (x)$ where max is defined on pseudoterms by $\prec$-induction:

- if $M \in$ Type, $\max (M)=\mathrm{SN}(M)$;

- if $M \in \operatorname{Proto}, \max (M)=(\lambda x: a(B) \cdot \max (M B))_{B \in \operatorname{cone}(M)}$;

- otherwise, $\max (M)=\{\emptyset\}$.

Then $(\rho, \zeta) \vDash \mathrm{FV}(M) \cup \mathrm{FV}(A)$. It follows from Proposition 30 that $M \in\langle\langle A\rangle\rangle_{\rho, \zeta}$. As $\left\langle\langle A\rangle_{\rho, \zeta} \subseteq \mathrm{SN}\right.$, we conclude.

\section{Applications of the main theorem}

\subsection{Strong normalisation results}

As stated in Corollary 11, Theorem 10 has several important consequences.

For $\mathcal{R}$-terminating extensions of the $\lambda$-cube, we know from [3] that subject reduction holds; so we are left to prove that the systems are stratified. To do so, notice that, if $M$ is a prototype, then $M: A$ with $A$ a kind and kinds are of the form ${ }^{7}$ :

$-*$,

- $\Pi x: A . B$ where $A$ and $B$ are kinds,

- $\Pi x: A . B$ where $B$ is a kind and $A$ is a type.

One can define a measure $\nu$ on kinds as follows:

$-\nu(*)=1$,

- $\nu(\Pi x: A \cdot B)=\nu(A)+\nu(B)+1$ if $A$ and $B$ are kinds,

- $\nu(\Pi x: A . B)=\nu(B)+1$ if $B$ is a kind and $A$ is a type.

Note that the measure is preserved by conversion. By uniqueness of types, this yields a measure $\mu$ on prototypes: define $\mu(M)=n$ if $\vdash M: A$ and $\nu(A)=n$ for some $A$. Extending $\mu$ to all pseudo-terms by letting $\mu(P)=0$ if $P \notin$ Proto, we obtain the following result. For every $P, Q$,

$$
P \prec Q \Rightarrow \mu(P)<\mu(Q)
$$

\footnotetext{
${ }^{7}$ Below we are implicitely assuming that algebraic data live in $*$ as in [3]; it is easy to adapt the proof to the other case.
} 
Hence the systems of the algebraic $\lambda$-cube are stratified. A similar technique applies to algebraic higher-order logic.

For $\mathcal{R}$-canonical and $\mathcal{R}$-left-linear extensions of the calculus of constructions with universes, the proof is more involved and requires a quasi-normalisation argument, as developed in [20]. The quasi-normalisation theorem shows that every type has a weak head normal form. This enables us to give a measure on types. As before, we can invoke uniqueness of types to turn this measure into a measure $\mu$ for prototypes with the property that $P \prec Q \Rightarrow \mu(P)<\mu(Q)$ for all pseudo-terms $P, Q$. Note that in this case it is crucial to know subject reduction and confluence of reduction on normal terms before the strong normalisation proof so we must restrict ourselves to confluent and left-linear rewriting systems. For such systems, the combined reduction is confluent on the set of pseudo-terms of the algebraic type system (this follows from [22]).

\subsection{Confluence results}

As noticed in [10], the combined reduction relation $\rightarrow_{\text {mix }}$ of an algebraic type system is in general not confluent on the set of pseudo-terms. However, it is straightforward to check that $\rightarrow_{m i x}$ is locally confluent on pseudo-terms. Using Newman's Lemma, one can lift Theorem 10 to $\mathcal{R}$-canonical algebraic type systems.

Proposition 32 Every $\mathcal{R}$-canonical algebraic type system with the subject reduction property is strongly normalising and confluent w.r.t. $\rightarrow_{m i x}$.

The results of Corollary 11 can all be lifted to $\mathcal{R}$-canonical algebraic type systems.

\section{Conclusion}

We have introduced in the unified framework of algebraic type systems a large class of algebraic-functional languages which includes all the systems considered in the literature so far. In this general framework, we have been able to address modularity questions. We have given a general criterion for algebraic type systems to be strongly normalising and shown that all the usual algebraic type systems satisfy this criterion. One nice aspect of the proof is that it gives a uniform treatment of all the usual algebraic type systems and emphasizes the fact that proving strong normalisation for algebraic type systems is not essentially more difficult than proving strong normalisation for pure type systems. It would be interesting to extend the present work to more powerful type systems: possible extensions to be considered are first-order inductive types (i.e. inductive types generated by first-order signatures, see for example [23]) or congruence types (an extension of algebraic type systems in which data come equipped with an elimination principle, see [8]). However, we feel more enclined to focus on two important problems which have remained unsolved so far: 
- subject reduction: it is an open problem whether algebraic type systems have subject reduction. This is a serious gap in the theory of algebraic type systems. Even for systems with subject reduction, such as the algebraic Calculus of Constructions, the situation is unsatisfactory because the proof of subject reduction is long and intricate. One possible approach to solve the problem would be to consider a labelled syntax for algebraic type systems in which all the usual properties of functional pure type systems (especially subject reduction, unicity of types and classification) hold and use these properties to prove strong normalisation of the labelled syntax (for stratified systems). Then, assuming the labelled syntax to be strongly normalising, one would transfer these results to the traditional syntax by proving the equivalence between the labelled and traditional syntaxes. This approach, introduced by T. Altenkirch to prove strong normalisation for the Calculus of Constructions with $\beta \eta$-reduction ([1]), is currently investigated by $\mathrm{P}-\mathrm{A}$. Mellies and the first author.

- modular proofs: our approach to prove strong normalisation is uniform in the sense that algebraic type systems are treated simultaneously with pure type systems. Yet in practice, one would like to know that an algebraic type system is strongly normalising if its underlying pure type system is. Note that such a result would require a purely syntactic proof as no assumption is made on the algebraic type system. See [7] for some preliminary work in this direction.

Another interesting direction for future research is to study the strength of the criterion for pure(and algebraic) type systems. Although every pure type system of interest is stratified, one can easily find pure type systems which are strongly normalising without being stratified. The easiest example is probably obtained by adding to the polymorphic $\lambda$-calculus a new sort $\triangle$ and an axiom $\triangle: *$. It would be instructive to compare our criterion with other strong normalisation criteria for pure type systems. It is easy to prove that any pure type system which can be embedded in the calculus of constructions with universes is stratified. The converse is not true: consider the pure type system with set of sorts $\mathbf{N}$ and with axioms $i+1: i$ (and no rules). This is a stratified pure type system, yet it cannot be embedded in the calculus of constructions with universes. However, we might hope that every stratified pure type system with finitely many sorts can be embedded in the calculus of constructions with universes.

\section{Acknowledgements}

Special thanks to the anonymous referees for suggesting significant improvements to the paper. This work was partially supported by the Esprit BRA project "TYPES" (Types for Proofs and Programs). 


\section{References}

1. T. Altenkirch. Constructions, inductive types and strong normalisation. $\mathrm{PhD}$ thesis, Laboratory for the Foundations of Computer Science, University of Edinburgh, 1994.

2. F. Barbanera and M. Fernandez. Combining first and higher order rewrite systems with type assignment systems. In M.Bezem and Groote [9], pages 60-74.

3. F. Barbanera, M. Fernandez, and H. Geuvers. Modularity of strong normalisation and confluence in the algebraic $\lambda$-cube. In Proceedings of LICS'94, pages 406-415. IEEE Press, 1994.

4. H.P. Barendregt. Lambda calculi with types. In S. Abramsky, D. M. Gabbay, and T.S.E. Maibaum, editors, Handbook of Logic in Computer Science, volume 2, pages 117-309. Oxford Science Publications, 1992.

5. G. Barthe. Combining dependent type theories with equational term-rewriting. Manuscript, 1995.

6. G. Barthe. $\eta$-reduction and algebraic rewriting in $\lambda$-calculus. Manuscript, 1995.

7. G. Barthe. Towards modular proofs of termination for algebraic type systems. Manuscript, submitted for publication, 1995.

8. G. Barthe and H. Geuvers. Congruence types. Presented at CSL'95. Submitted for publication in the proceedings, 1995.

9. M. Bezem and J-F. Groote, editors. Proceedings of TLCA'93, volume 664 of Lecture Notes in Computer Science. Springer-Verlag, 1993.

10. V. Breazu-Tannen. Combining algebra and higher-order types. In Proceedings of LICS'88, pages 82-90. IEEE Press, 1988.

11. V. Breazu-Tannen and J. Gallier. Polymorphic rewriting conserves algebraic strong normalisation. Theoretical Computer Science, 83:3-28, 1990.

12. M. Fernandez. Modèles de calcul multiparadigmes fondés sur la réécriture. $\mathrm{PhD}$ thesis, Université Paris-Sud Orsay, 1993.

13. M. Fernandez and J-P.Jouannaud. Modularity of termination of term-rewriting systems revisited. In Recent Trends in Data Type Specification, volume 906 of Lecture Notes in Computer Science, pages 255-272. Springer-Verlag, 1994.

14. J. Gallier. On Girard's "candidats de réducibilité". In P. Odifreddi, editor, Logic and Computer Science, pages 123-203. Academic Press, 1990.

15. H. Geuvers and M.J. Nederhof. A modular proof of Strong Normalization for the Calculus of Constructions, Journal of Functional Programming 1, 2 (1991), 155189.

16. H. Geuvers. Logics and type systems. $\mathrm{PhD}$ thesis, University of Nijmegen, 1993.

17. H. Geuvers. A short and flexible proof of strong normalisation for the calculus of constructions. In P. Dybjer, B. Nordström, and J. Smith, editors, Proceedings of TYPES'94, volume 996 of Lecture Notes in Computer Science, pages 14-38. Springer-Verlag, 1995.

18. J-Y. Girard. Interprétation fonctionelle et élimination des coupures dans l'arithmétique d'ordre supérieur. $\mathrm{PhD}$ thesis, Université Paris 7, 1972.

19. J-P. Jouannaud and M. Okada. Executable higher-order algebraic specification languages. In Proceedings of LICS'91, pages 350-361. IEEE Press, 1991.

20. Z. Luo. Computation and Reasoning: A Type Theory for Computer Science. Number 11 in International Series of Monographs on Computer Science. Oxford University Press, 1994.

21. A. Middeldorp. Modular properties of term-rewriting systems. $\mathrm{PhD}$ thesis, Department of Computer Science, Vrije Universiteit, Amsterdam, 1990. 
22. F. Müller. Confluence of the lambda calculus with left-linear algebraic rewriting. Information Processing Letters, 41:293-299, 1992.

23. C. Paulin-Mohring. Inductive definitions in the system Coq. Rules and properties. In Bezem and Groote [9], pages 328-345.

24. W. Tait. A realisability interpretation of the theory of species. In R. Parikh, editor, Logic Colloquium 73, volume 453 of Lectures Notes in Mathematics, pages 240-251, 1975.

25. J. Terlouw. Strong normalisation in type systems: a model-theoretical approach. In Dirk van Dalen Festschrift, pages 161-190. University of Utrecht, 1993. To appear in Annals of Pure and Applied Logic.

26. Y. Toyama. On the Church-Rosser property for the direct sum of term rewriting systems. Journal of the ACM, 34(1):128-143, 1987. 